\title{
Knowledge, Attitude and Practice of Exclusive Breast Feeding among Mothers: A case study of Sanepa Sachal, Lalitpur District
}

K.C.Ganga, Ph.D.

\begin{abstract}
Breastfeeding has been accepted as the most vital intervention for reducing infant mortality, ensuring optimal growth and development of children. Proper breastfeeding provides all essential nutrients for the first 6 months and is one of the cost effective intervention for reducing infant mortality and disease. It is one of the essential actions for infant development and survival. Maternal knowledge and attitudes as well as socio-demographic and cultural factors influence the practice of exclusive breastfeeding (EBF).

This study reflects the knowledge, attitude and practice of EBF among breastfeeding mothers with infants aged 0-6 months. Exclusive breastfeeding means feeding a baby with only mother's breast milk without any additional food or drinks including water. The main objective of this research is to explore the knowledge, attitude and practice of EBF behavior of the mothers. Using the simple random sampling, Sachal area, ward no.2 of Lalitpur metropolitan city was selected as study area. For the data collection 30-breastfeeding mother-infant pairs were purposively selected as the key informant. Data were collected from 30 mother-infant pairs by using interview schedules.
\end{abstract}

Keywords: knowledge, attitude, practice, exclusive breastfeeding, breast milk.

\section{Introduction}

Exclusive breast-feeding is a common practice among the mothers in rural areas. Proper breastfeeding provides all essential nutrients for the first 6 months and it is one of the cost effective intervention for reducing infant mortality and disease. "Exclusive breastfeeding" is defined as no other food or drink, not even water, except breast milk (including milk expressed or from a wet nurse) for 6 months of life, but allows the infant to receive ORS, drops and syrups (vitamins, minerals and medicines) (WHO,2011). Breastfeeding has been accepted as the most vital method to prevent infant mortality. Obviously, breastfeeding has been going on since mammals existed on Earth. It has been in practice through different methods in different era. If we go through history of mankind, before invention of easy alternatives, breastfeeding has been only option. Breastfeeding practice rates differ in different communities as well as in different countries. 
As quoted by Subba et.al, (2007) at present, 11\% of under 5 year children in Nepal are underweight and 29\% are wasted (Ministry of Health and Population [MOHP] [Nepal], New ERA, and ICF International Inc., 2012). A study of infant feeding practices of mothers in an urban area of Pokhara, Nepal revealed that among 168 mothers interviewed, prevalence of breastfeeding was 99.4\% (167). Only 43.5\% of the mothers initiated breastfeeding within one hour of birth and $60.5 \%$ were practicing exclusive breastfeeding at 5 months.

The infant mortality rate (IMR) of Nepal in 2019 was 27.022 deaths per 1000 live births (NDHS, 2019). Globally, an estimated 1.3 million lives are lost each year due to inadequate exclusive breast-feeding (EBF). Less than $35 \%$ of infants worldwide are exclusively breastfed during the first six months of life. Furthermore, malnutrition is responsible for one-third of the 8.8 million deaths annually among children under five. Over two-third of these deaths are often associated with inappropriate feeding practices such as bottle-feeding. More than 15\% of 24 lakh child deaths could be averted in India by optimal breastfeeding practices. Breastfeeding is the ideal method suited for the physiological and psychological needs of an infant (Ekambaram et.al., 2010). The mother's antibody is in the breast milk provides immunity to infants to protect from different diseases (WHO, 2007).

Breast milk is life, containing all the nutrients vital for nourishment, as well as growth factors believed to help in tissue development and antibodies to fend of infections. It is always at the right temperature, requires no mixing, sterilization or equipment and is safe regardless of the quality and availability of water. The percentage of nutrients differs in every feeding and even within feedings, and the amount is triggered by the mother's hormonal response to the needs of the baby. Breast feeding promotes bonding between mothers and baby and prevents conception (UNICEF, 1990).

The role of lactation in establishing social relationships is not peculiar to humans. The social dimension of human breastfeeding has its precursors in our recent evolutionary heritage. In our own species infant nutrition, central to early socialization, comprises an ideal vehicle for exploitation in multiple symbolic contexts. One illustration of this is the tendency in various societies for non-maternal nursing to contribute to alternative social structures in the form of "milk kinship" (Parkes, 2004). Exclusive breastfeeding in the first six months of life stimulates babies' immune systems and protects them from diarrhea and acute respiratory infections (UNICEF, 2006). Exclusive breastfeeding for the first six months of life is now considered as a global public heath goal that is linked to reduction of infant morbidity and mortality, especially in the developing world (WHO, 2011).

The world health organization (WHO) recommends exclusive breast feeding (EBF) for the first six months of life while it is advised to provide adequate and safe complementary foods with breast feeding for up to two years and beyond. EBF remains 
uncommon in most countries (both developed and developing), even in countries with high rates of breastfeeding initiation. EBF rates in infants less than six months of age varied from as low as $20 \%$ in central and eastern European countries to $44 \%$ in south Asia (Imdad et.al., 2011).

There is high quality evidence that infant not exclusively breastfed are at increased risk of death from diarrhoea, pneumonia and neonatal sepsis. It is proposed that exclusive breastfeeding for 6 months, followed by continued breastfeeding to 12 months, could prevent 1,301,000 deaths or 13 percent of all child deaths under 5 years in a hypothetical year (Annetteand Lisa 2007).The NDHS 2011 report of Nepal states “In Nepal children born in the five years preceding the survey (98\%) were breastfed at some time. However, contrary to World Health Organization's (WHO) recommendations, only around half of the children under six months (53\%) are exclusively breastfed.”

Nepal being a developing country, we have higher infant mortality rate. Such a tremendous case will definitely leave its impacts in other sectors of life. One of the vital causes for high infant mortality is undoubtedly being the improper breastfeeding practice; it is essential in Nepal to make people aware about the importance of exclusive breast-feeding as recommended by WHO standard.

\section{Objective and Methods}

The key objective of this research was to explore the knowledge, attitude and practice of exclusive breast-feeding and to identify the affecting factors, which influence mothers' breast-feeding behavior.

It is a community based study, which was designed to explore practice, knowledge and attitude to breastfeeding practice and to assess factors associated with breastfeeding among women who had infants aged between 1-12 months. This study primarily focuses its deeds to determine a qualitative relationship between two fundamental parametersknowledge, attitude and practices of EBF and affecting factors, which influence mother's breast-feeding behavior. The study was conducted in Sachal area; ward no. 2, of Lalitpur metropolitan city. For this 30-breastfeeding mother-infant pairs were selected as respondent using the simple random sampling technique.

Employing both descriptive and exploratory research design this research was carried out. The exploratory research design was used to explore the attitude regarding exclusive breast-feeding (EBF), where the descriptive research design was implemented to describe various information related to exclusive breast-feeding. For this, necessary primary information was collected from the field. Primarily the qualitative information is used in this study, which is gathered through the fieldwork. 


\section{Discussion on findings}

Result of data analysis is presented in this section, which covers socio-demographic information of respondents, knowledge of breastfeeding mothers, attitude of breastfeeding mothers and practice of exclusive breastfeeding. Socio-demographic characteristics of the respondents was presented in Table 1, mother's knowledge about exclusive breastfeeding is presented in Table 2, likewise Table 3 talks about mother's attitude of Exclusive breastfeeding and in Table 4 discusses the practice of exclusive breastfeeding.

\section{Socio-demographic characteristics}

Breast-feeding behavior in human has been influenced by various sociodemographic and cultural factors. In health related issues, proper knowledge is an essential factor; however, there are environmental, socio-cultural, socio-economic, demographic and other human factors that may create deviations on knowledge, attitude, and practices of mother on her breast feeding behavior. Breast-feeding is socially constructed and controlled practice. So, different social groups, caste/ethnicity, religion, culture, traditional practices directly and indirectly affect knowledge, attitude and practices of exclusive breastfeeding. If all the social systems promote and practices exclusive breast-feeding (EBF), all the infants shall become healthy and society saves time and money for childhood diseases. Knowledge, attitude and practice of exclusive breast-feeding are properly described here.

Exclusive breastfeeding not only carries an importance in health issues but it also carried social responsibilities within. If a mother is educated and informed about breastfeeding, then she can perform it well and which gives healthy offsprings that can create a healthier society. Informed mother will also pass on same knowledge to her coming generation so practice will be continued further. As Nepali community and particularly Newar community being male dominated community, sometimes there is a difference in a way a boy or girl raised, which starts from very beginning of their life. These also can make impact on their health, social status and also can affect in their psychology. So, lack of knowledge and practice is as important issue as breastfeeding. According to the findings of the study discussed in table 1 , out of 30 breastfeeding respondents, 26 (86.67 \%) were Newar, 3 (10 \%) were Kshetri and 1 (3.33\%) Magar in ethnicity.

Mother's occupation directly affects the exclusive breast-feeding. In spite of their knowledge, most of the educated mothers are not able to care their infants because of the nature of the job. This means EBF practice is influenced by the nature of mother's work. For example in Nepal the government grants only 90 days of maternity leave to the maternal mothers. While they return back to job after the leave, they do not have time to care their infant therefore they are forced to escape breast-feeding on their office time. In spite of all these, most of the respondents were found inadequately practicing exclusive breastfeeding.This research shows that none of the mothers involved in service practiced 
exclusive breast-feeding. Mothers who were involved in petty business were able to breastfeed more than service holder mothers. Likewise 25 (83.3\%) mothers were housewives following 2 (6.7\%) were government service holder and 3 (10.0\%) were manual worker by occupation.Among the total 30 breast-feeding mothers most of them, 21 (70\%) were 20-25 years old. Likewise, 5 mothers (16.5\%) were 25-30 years old following 2 (6.6\%) were below 20 and 2 (6.6\%) were above 30 years old.

\section{Table 1: Ethnicity, age, and occupation of breastfeeding mother's in the study area}

\begin{tabular}{|l|l|l|}
\hline Social characteristics & Number & Percentage \\
\hline Ethnicity of family & & \\
Newar & 26 & 86.7 \\
Kshetri & 3 & 10.0 \\
Magar & 1 & 3.3 \\
\hline Total & 30 & 100.0 \\
\hline Mother's occupation & & \\
Housewives & 25 & 83.3 \\
Government service & 2 & 6.7 \\
Manual work & 3 & 10.0 \\
\hline Total & 30 & 100.0 \\
\hline Mother's age & & \\
Below 20 & 2 & 6.7 \\
20-25 & 21 & 70.0 \\
25-30 & 5 & 16.6 \\
Above 30 & 2 & 6.7 \\
\hline Total & 30 & 100.0 \\
\hline
\end{tabular}

Source: Field Study, 2019.

\section{Family structure and education of the respondents}

Family structure and education also plays significant role in exclusive breast-feeding practice of mother. Family structure also has very important impact on understanding issues in family members. Various studies show that the knowledge of breastfeeding depends on the respective family structure. Mothers living in joint family have better chances of sharing knowledge with family members. This study reported that, out of 30 respondents 21 (70 \%) respondentsbelonging to joint family were found having good knowledge about EBF whereas only 9 (30\%) mothers were living in nuclear family who had not fair knowledge of EBF. 
Patan Pragya (Volume: 6, Number: 1 2020)

Table 2: Family structure and education of breast-feeding mother in the study area

\begin{tabular}{|l|l|l|}
\hline Family structure & Number & Percentage \\
\hline Nuclear & 9 & 30.0 \\
Joint & 21 & 70.0 \\
\hline Total & 30 & 100.00 \\
\hline Mother's education & & \\
Literate & 8 & 26.7 \\
Primary & 7 & 23.3 \\
Secondary & 13 & 43.3 \\
Bachelor & 2 & 6.7 \\
\hline Total & 30 & 100.0 \\
\hline
\end{tabular}

Source: Field Study, 2019.

Education plays a vital role in every aspect of life including exclusive breastfeeding. The outcome of this study shows that most of the respondents 13 (43.3\%) received the secondary level education, only 2 (6.7\%) respondents obtained bachelor level following literate respondents were only 8 (26.7\%) and 7 (23.3\%) respondents were in the category of primary education.

\section{Knowledge of Exclusive Breast-feeding}

Personal knowledge is rooted in particular experience and involves intangible factors, such as personal beliefs, perspective, and the value system. Silent knowledge is hard to articulate with formal language but not impossible. It contains subjective insights, and feelings. The knowledge, as already stated, is the combination of how people came to know and how they perceive the information. This study was conducted to identify the proper knowledge about the definition of exclusive breast-feeding.

In Nepal breast-feeding has special cultural value. Being a developing country, the rate of increase in infant mortality rate is higher and it is very important in Nepal to make people aware about the importance of exclusive breast-feeding till six months. Shrestha (2054) had studied 50 breastfeeding mothers regarding knowledge, attitude and practice and its outcomes about exclusive breastfeeding showing that only $8 \%$ mothers of literate group knew about EBF. Hundred percent illiterate mothers did not have knowledge about exclusive breast-feeding. And there was no significant difference between ethnic group, types of family, age of mother and occupation among literate and illiterate mothers about knowledge, attitude, and practices of breast-feeding.

According to this research, 22 (73.33\%) respondents thought that exclusive breastfeeding $(\mathrm{EBF})$ is breast feeding without giving other food, liquid, or water, 3 respondents (10.0\%) thought that EBF is breast feeding with water plus, 3 (10.0\%) thought breast 
feeding with other milk, 1(3.33\%) thought breast-feeding with complementary food, and the remaining 1(3.33\%) has no idea about the question. Only 22(73.33\%) can define Exclusive Breast Feeding (EBF) properly as per World Health Organization (WHO) recommendation. Similarly, findings of the study show that only 15(50.0\%) children are adequately breastfed, among them 8 (26.7\%) breastfeeding mother have good knowledge, 5(16.6\%) have fair knowledge and $2(6.7 \%)$ have no knowledge about exclusive breast-feeding.

Breastfeeding should ideally start soon after the baby is born. A baby is usually alert after birth and will spontaneously seek the breast if left undisturbed in skin-to-skin contact with the mother's body. Various researches suggest that a mother should allow her baby to feed when the baby shows a sign. Among the 30 respondents, 15(50.0\%) mothers think that the best time to start breast feeding after birth is within one hour, 9 (30.0\%) mothers think that it should be between 1- 4 hour, 3 (10.0\%) think its 5-12 hours and $3(10.0 \%)$ has no idea about this.

The first 24 hours of a baby's life is crucial to a positive breastfeeding experience for both mother and baby. Newborn babies are very alert for the first two hours after birth and, typically, are eager to feed. Because they have tiny appetite, newborn will need to feed frequently. Intervals of up to 8 hours between feeds were not associated with excessively low blood glucose concentrations in newborns (WHO, 2011). Thisstudy found that majority of the breast feeding mother 14(47\%) respondents thought that it should be repeated every one to two hours on day time and $13(43 \%)$ respondents thought that it should be repeated on every two to three hours at night time but 3 (10\%) mothers had no idea.

Breastfeeding itself is very important for child's health. It cannot be denied that exclusive breastfeeding have many health benefits for a baby.The research shows the respondents' opinion about the advantages of exclusive breast-feeding to mother's health. Majority of the breast-feeding mothers were aware about the advantages of exclusive breastfeeding. Based on the data of this study 24 (80.0\%) mothers were in favor of less risk to breast cancer whereas 3 (10.0\%) mothers for natural contraceptive device, 2 (6.67\%) mothersbelieved less risk of ovarian cancer and weight loss.Likewise, 1 (3.33\%) respondentthought that EBF is for mother's satisfaction, mother-baby relation and prevention from breast engorgement. 
Patan Pragya (Volume: 6, Number: 1 2020)

Table 3: mother's knowledge about Exclusive Breastfeeding

\begin{tabular}{|l|l|l|}
\hline Characteristics & Number & Percentage \\
\hline Understanding of EBF & & \\
Breastfeeding without giving other liquid food & 22 & 73.4 \\
Breastfeeding with water plus & 3 & 10.0 \\
Breastfeeding with other milk & 3 & 10.0 \\
Breast feeding with complimentary food & 1 & 3.3 \\
No idea & 1 & 3.3 \\
\hline Time of First breastfeeding after birth & & \\
Within one hour & 15 & 50.0 \\
1-4 hour & 9 & 30.0 \\
5-12 hour & 3 & 10.0 \\
No idea & 3 & 10.0 \\
\hline Knowledge on time interval of EBF & & \\
1-2 hours (day time) & 14 & 47.0 \\
2-3 hours (night time) & 13 & 43.0 \\
No idea & 3 & 10.0 \\
\hline Knowledge on advantage of EBF & & \\
Less risk of breast cancer & 24 & 80.0 \\
Natural contraceptive & 3 & 10.0 \\
Less risk of ovarian cancer & 2 & 6.7 \\
Mother's satisfaction & 1 & 3.3 \\
\hline Role of mother's education in EBF & & \\
Having good knowledge & 20 & 66.7 \\
Having fair knowledge & 10 & 33.3 \\
\hline
\end{tabular}

Source: Field Study, 2019

Mother's education was found to be directly related with her knowledge of exclusive breast-feeding. Mothers with higher education (above SLC) were found to know well regarding EBF. Those mothers, who were involved in service, have good knowledge about EBF. Like mother's education, occupation of a mother also clearly impacts on how she raises her child. It clearly shows that the mothers who are in contact with educated circle acquire better knowledge than the housewives and those involved in daily wages. Table 3 shows that among 30 respondents, 20 (66.67 \%) have good knowledge about exclusive breast-feeding whereas 10 (33.33\%) have only fair knowledge.

\section{Mother's attitude on Exclusive Breastfeeding}

Attitude is manner, nature, feeling, position, etc. with regard to a person or thing, tendency or orientation, especially of the mind. It is the understanding derived from various Anthropological, sociological, cultural, ethnic, educational, and perceptual background. It has been realized that even knowledge and education are not enough for mothers to manage breastfeeding. There is a need to understand the mothers' belief patterns about breastfeeding followed by interventions to modify their beliefs, practices and their responsibilities. 
The mother and child as a unit have a right to breastfeed. It is not the unconditional right of the child to breastfeed, or that would oblige the mother to breastfeed regardless of her own situation. The right to breastfeed means that no outside party has the right to interfere with the relationship between the mother and child.

Breast-feeding can greatly reduce the feeding expenses of baby. However the breastfeeding mother needs some additional diet for which it needs some additional expenses but it is essential for her health. According to the research, it was found that, 29 (96.67 \%) mothers agreed that breast-feeding could reduce family expenses. Only 1 (3.33 \%) mother had strong positive opinion about this. Nobody argued on this issue. As great as breast-feeding has it's benefits, it even easier if it is done correctly. It protects from lot of other hassles like preparing bottle, and caring about hygiene. Breastfeeding is ready anytime and anywhere. According to the findings of this study, table 4explained thatmajority of mothers 29 (9.67\%) agreed that breastfeeding is very easier than using infant formula and 1 (3.33 \%) strongly believes it.

Table 4: mother's attitude on Exclusive Breast Feeding

\begin{tabular}{|l|l|l|}
\hline Characteristics & Number & Percentage \\
\hline Reduce family expenses & 29 & 96.67 \\
Having strong positive opinion of EBF & 1 & 3.33 \\
Easier than infant formula & 29 & 96.67 \\
Strong belief & 1 & 3.33 \\
\hline
\end{tabular}

Source: Field Study, 2019.

A prelacteal food is any food except mother's milk provided to a newborn before initiating breastfeeding. Prelacteal feeding is a major barrier to exclusive breastfeeding. It is a prevalent practice in Nepal. Little is known about the factors associated with providing prelacteal feeds to the Nepalese newborn (NDHS, 2011).According to the findings of this research, 28 (93.33 \%) mothers agreed to the fact that prelacteal food is harmful for babies whereas 2 (6.67\%) mothers disagreed with the fact. Expressed breast milk is also safe for the baby, if mother do not have time to breast feed to her baby because of workload and other reason. Almost all mothers 28 (93.33 \%) disagreed on it.

Nepal Government and most of other organizations give only 90 days maternity leave to childbearing mothers. It may be the barrier for exclusive breast-feeding up to six months of baby's life. So that Maternity leave is not enough to successful breast-feeding. Among the 30 respondents 11 (36.67\%) mothers have no idea, 7 (23.33\%) mothers agree with government policy and 12 (40\%) breastfeeding mothers disagree with government policy.

In Nepal there is no any knowledge and practice about expressing breast milk. Expressing milk means squeezing milk out of breast so we can store it and feed it to the 
baby at a later time. We can do this by using hands or pumps. Mothers expressed breast milk is safe and this can be useful if the breast-feeding mother has to leave her infant due to various reasons. Nearly all 93 percent mothers disagreed that expressed breast milk is safe; 2 mothers had no idea about this.

\section{Practice of Exclusive Breast-Feeding}

In present context of Nepal, practice of institution delivery is increasing because of awareness, financial support, and encouragement provided by the government. Based on the findings, this study all 30 (100.0\%) mothers delivered at health institution. Mothers who delivered at hospitals and health institutions were more likely to practice exclusive breastfeeding. This is so because mothers who delivered at health institutions could have more chance to receive obstetric and postnatal care, nutritional education and counseling on the benefits of breastfeeding, correct positioning and attachments of mother and baby. Exclusive breastfeeding in the study area is considerably related with breastfeeding counseling after delivery. Besides, pregnancy and childbirth are still perceived as natural phenomena, not requiring formal health services (UNICEF 1990).Initiation of breast-feeding within one hour after birth reduces postnatal bleeding, increases flow of breast milk and prevents neonatal death. Most of the people think breast milk does not come immediately after birth. So, Nepalese community has a practice of giving first breast milk of other female (Dudh Khwaune Ama/dhai ama).

Colostrum is the breast milk that women produce in the first few days after delivery. It is thick and yellowish or clear in color. It has antibody, which protects baby from many diseases. In our traditional practice, breast is squeezed until white milk comes because it is thought that first thick milk is dirty and produces indigestion problem and diarrhea. This research reported that all 30 (100.0\%) respondents who were basically breastfeeding mothers fed colostrum milk to their babies. The respondents who had deliveredat Hospitals were got Breast-feeding counseling after delivery. Among them 2 (6.7\%) mothers thought that baby could not digest, 2 mothers with babies were premature and one mother had got her relatives advised her not to feed. It indicates that people have practiced to feed colostrum milk and accepted scientific knowledge.

This research shows that exclusive breastfeeding practice according to world health organization (WHO) recommendation. According to the findings of this study, 3(10.0\%) mothers exclusively breast fed their baby for less than two months, 5 (16.67 \%) mothers fed for 2-3 months, and 9 (30 \%) mothers exclusively breast fed for 4-5 months. Only 13 (43.33 \%) mothers had exclusively breast fed up to 6 months to their baby as per World Health Organization (WHO) recommendation. 
Patan Pragya (Volume: 6, Number: 1 2020)

Table 5: Practice of Exclusive Breast Feeding

\begin{tabular}{|l|l|l|}
\hline Characteristics & Number & Percentage \\
\hline $\begin{array}{l}\text { Breast feeding counseling after delivery } \\
\text { Yes } \\
\text { No }\end{array}$ & 30 & 100.0 \\
\hline Feeding colostrum milk & -- & \\
\hline Duration of EBF & 30 & 100.0 \\
Less than 2 months & 3 & \\
2-3 months & 5 & 10.0 \\
4-5 months & 9 & 16.7 \\
Up to 6 months & 13 & 30.0 \\
\hline Attendant of the delivery & 00 & 43.3 \\
Health professional & 30 & 100.0 \\
Relative, family member & -- & -- \\
\hline Place of delivery & 30 & 100 \\
Government Hospital & -- & -- \\
Private hospital & \multicolumn{2}{|l}{} \\
\hline
\end{tabular}

Source: Field Study, 2019.

Above table 4 discusses that at the time of hospital delivery, out of 30 all (100\%) respondents had got delivery service by health professional attendant. This indicates that all the respondents had got skilled delivery service.This research did not notice any sex discrimination on EBF practice up to six months of baby although most of the female babies get solid diet after the completion of 5 months and male baby get solid diet after the completion of 6 due to their culture. So comparatively male children were exclusively breastfed.Despite having fair or no knowledge about breastfeeding, many mothers could and could not exclusively breastfeed their children for various reasons. This study shows inarticulate positive relationship between knowledge and practice of exclusive breastfeeding. However, mothers having inadequate or no knowledge of exclusive breast-feeding were also found adequately practicing exclusive breast-feeding.

\section{Conclusion}

The findings of this research show the relation between knowledge, attitude and practices prevalent regarding the breast-feeding practices. People having knowledge in subject do not mean they keep same defiance and drill it in their life. Knowledge received sometimes does not match with that attitude they have or carry because so many things like society, media and others influence them. Same knowledge sometimes cannot be implemented because many factors affect in this.

This research Knowledge, attitude and practice of exclusive breastfeeding in Sachal, ward no.2, Lalitpur metropolitan city gave the same idea. Factors like Occupation and health condition arebarriers for a mother, which stops her from practicing exclusive breast-feeding. In 
this study, I also found out that, even conservative society of Sachal areais changing with time and adapting new concept and culture. It could be because of exposure of media and information to new generation. Some of them are able to convince old generation to practice this.

\section{References}

Annette, Beasley \&Lisa, H Amir.(2007). Infant Feeding Poverty and Human Development.International Breastfeeding Journal Oct 20072:14.Doi: 10.1186 / 1746-4358-214, Retrieved Oct 15, 2013 http://by132w.bay132.mail.live.com.

Ekambaram, M., Bhat, V., \& Ahamed, M. A. P. (2010).Knowledge, attitiude and practice of breastfeeding among postnatal mothers. Current Pediatric Research, 14(2), p,119.

Imdad.A, M. Y. Yakoob, \&Z. A. Bhutta. (2011). "Effect of breastfeeding promotion interventions on breastfeeding rates, with special focus on developing countries," BMC Public Health, vol. 11, no. 3, article S24, 2011.View at: Publisher Site | Google Scholar

NDHS.(2011). Ministry of Health and Population, Nepal, New ERA, and ICF International Inc. (2012).Nepal Demographic and Health Survey 2011. Kathmandu, Nepal.

NDHS.(2016). Ministry of Health and Population, Nepal, New ERA, and ICF International Inc. (2017).Nepal Demographic and Health Survey 2016. Kathmandu, Nepal.

Parkes, P. (2004). Milk kinship in Southeast Europe: Alternative social structures and foster relations in the Caucasus and the Balkans.Soc. Anthropology., 12: 341-58.

Shrestha, Khadaga Laxmi. (2054). A comparative study of knowledge, attitude and practice about exclusive breast feeding among literate and illiterate mother and its out come up to 4-6 months baby (Child) in Kanti Hospital M.C.H. Clinic.

Retrieved Jan 6, 2013 http://www.hellisnepal.gov.np/Journal_thesis.

Subba, S. H., Chandrashekhar, T. S., Binu, V. S., Joshi, H. S., Rana, M. S., \& Dixit, S. B. (2007). Infant Feeding Practices of Mothers in an Urban Area in Nepal.Kathmandu Univ Med J, 5(1), 42-7.

UNICEF. (2006). Proress of children-exclusive breast Feeding: a report card on nutrition: number 4, May 2006.

UNICEF. (1990). Innocenti Declaration: On the Protection, Promotion and Support of Breastfeeding, UNICEF Nutrition Cluster (H-8F), 3 United Nations Plaza,

World Health Organization (WHO). (2011).The optimal duration of exclusive breastfeeding: report of an expert consultation. Geneva: World Health Organization, Department of nutrition for health and development and department of child and adolescent health and development; 2011.

World Health Organization.(2007).Protein and amino acid requirements in human nutrition.World health organization technical report series, (935), 1. 